\title{
From the journals
}

\section{Prevention of diabetes in south Asians}

Increased prevalence of type 2 diabetes in south Asians is observed in many parts of the world including the UK. South Asians in the UK have the highest death rate from coronary heart disease. Much of this increased risk is attributed to the increased risk of type 2 diabetes. The risk of type 2 diabetes in south Asians is four times that of Europeans. On average, they develop diabetes about 10 years earlier than Europeans. The increased incidence of type 2 diabetes is strongly associated with rising central obesity and hyperinsulinaemia. The relationship between obesity and hyperinsulinaemia may occur at lower levels of fatness in south Asians compared to Europeans. In recognition of this, a body mass index(BMI) of higher than $23 \mathrm{~kg} / \mathrm{m}^{2}$, is classed as overweight in south Asians.

Recently, the "Diabetes Prevention Programme" studied 3234 patients with impaired glucose tolerance and compared it with the effects of intense lifestyle intervention, treatment with metformin, and routine care. Over five years, the risk of diabetes was reduced by $58 \%$ in the lifestyle intervention group and $31 \%$ in the metformin group. Although prevention of diabetes should be of central concern, south Asians with diabetes should be subjected to lower thresholds for intervention in high blood pressure, dyslipidaemia or hyperglycaemia compared to Europeans. British Medical Journal 2003; 327: 1059-60.

\section{Use of herbal medicines in children}

The incidence of toxicity in relation to herbal drug therapy is unknown. The toxicity of herbal medicines may be due to intrinsically toxic constituents of the herbal ingredients. Herbal medicines may cause allergic reactions and may interact with prescribed or over the counter medicines. The quality of herbal medicines need improvement. When herbal medicines are given to children, parents should obtain these from reliable sources. Health professionals should remember that many plant-derived chemicals are the basis of conventional drug therapies and therefore, be non-judgmental on parents using herbal medicines for their children. Archives of Diseases in Childhood 2003; 88: 1032-3

\section{Probiotics for preterm infants}

Healthy infants develop a colonising microflora which is dominated in the bowel by non-pathogenic species. Preterm infants in intensive care units develop an abnormal pattern of bowel colonisation compared to healthy infants and children. The use of antibiotics and infection control procedures limits the extent to which preterm infants are exposed to normal commensal microorganisms. In these infants the bowel becomes a major reservoir for antibiotic resistant bacteria. The abnormal pattern of colonisation in preterm infants may also contribute to the pathogenesis of neonatal necrotising enterocolitis (NEC).

One way to encourage bowel colonisation with desirable flora is through administration of probiotic bacteria. Probiotic bacteria are defined as live microbial supplements that colonise the gut and provide benefit to the host. Use of probiotics could lead to improvement in nutrition, reduction in the incidence of sepsis, use of antibiotics, and prevention of neonatal NEC.

Currently, many different preparations containing live probiotic bacteria are available as food stuffs from commercial outlets. Probiotics are considered to be food and are not subject to the controls that are applied to licensed drugs. The ideal characteristics of probiotic strains to be used in preterm infants have yet to be defined. Probiotics may offer potential benefits for preterm infants, but there is a need for clinical trials of sufficient size to allow clinically important outcomes to be investigated. Archives of Diseases in Childhood. Foetal and neonatal edition 2003; 88: F354

\section{Minimising contrast agent-induced nephropathy following cardiovascular procedures}

Nephropathy induced by radiographic contrast is a serious and potentially fatal complication. The risk can be minimised by careful selection of patients and attention to details during the procedure itself. The procedure should be tailored to the patient's overall clinical condition. This would minimise the amount of contrast agents administered and the dose of X-rays used. Although there is no dose of contrast agent that is completely safe, studies have indicated that for patients at substantial risk, severe nephropathy is uncommon unless the dose of the contrast agent exceeds $100 \mathrm{ml}$. The cardiologist planning a procedure is responsible for assessing the patient's risk and for formulating a strategy to minimise the amount of contrast agent needed. New England Journal of Medicine 2003; 349: 1311-2. 


\section{Intravenous magnesium in asthma: current recommendations}

The current British Guideline on the management of asthma suggests use of a single intravenous dose of magnesium sulphate for the treatment of patients with acute asthma, life-threatening asthma, or near fatal asthma. This unlicensed use of the drug is not justified, because the data available is weak and conflicting. Evidence from well designed randomised controlled trials is needed to confirm the effects of parenteral magnesium in acute asthma, to determine which patients are likely to benefit, and its place relative to alternative treatments. Drug and Therapeutic Bulletin 2003; 41: 79-80

\section{Paroxetine: not effective in children and adolescents}

The Committee on Safety of Medicines (CSM) advises against the use of paroxetine in children and adolescents under the age of 18 years to treat depressive illness. New data from clinical trials has shown that paroxetine is ineffective and harmful in this age group. The drug should be gradually tapered off in present users to avoid symptoms of discontinuation. WHO Pharmaceuticals Newsletter 2003; 4: 2

\section{Management of constipation: behavioural treatment}

For many patients, laxatives do not provide sustained relief of symptoms. When traditional treatments have failed, behaviour therapy has been shown to be effective. This includes habit training and teaching the patient to normalise pelvic floor function (to help sphincter activity) and psychological support. This mode of treatment has been effective in patients with slow gut transit, impaired rectal emptying and in patients with constipation after childbirth or pelvic surgery. British Medical Journal 2003; 327: 459-60

Kusum de Abrew, Senior Lecturer in Pharmacology, Faculty of Medicine, University of Colombo, Sri Lanka. e-mail: 271002@celltelnet.lk

\section{Money, morals and the conquest of mortality (2)}

Here, then, is the message from ethics; a call for action rather than an appeal to theory. It's time to look at the society in which we live, and to ask ourselves "Is this a society in which there's real justice? Is this a society where I and my loved ones, in our time of trouble, can be sure to access care which is compassionate, thoughtful and appropriate?" I don't know what conclusions you'll reach in this Summit, but I do know that you'll have wasted your time, and failed the constituency of the ill, if you fail to think deeply about these questions, and to suggest plans of action which will let us answer "yes" to both.

Miles Little. Keynote address at the Australian Health Care Summit 2003. Medical Journal of Australia. 2003; 179: 432 